\title{
Article \\ Fractal Dimension Analysis to Detect the Progress of Cancer Using Transmission Optical Microscopy
}

\author{
Liam Elkington, Prakash Adhikari (D) and Prabhakar Pradhan *(D)
}

check for

updates

Citation: Elkington, L.; Adhikari, P.; Pradhan, P. Fractal Dimension Analysis to Detect the Progress of Cancer Using Transmission Optical Microscopy. Biophysica 2022, 2, 59-69. https://doi.org/10.3390/

biophysica2010005

Academic Editors: Ricardo L. Mancera, Paul C Whitford and Chandra Kothapalli

Received: 29 November 2021

Accepted: 4 January 2022

Published: 7 January 2022

Publisher's Note: MDPI stays neutral with regard to jurisdictional claims in published maps and institutional affiliations.

Copyright: () 2022 by the authors Licensee MDPI, Basel, Switzerland. This article is an open access article distributed under the terms and conditions of the Creative Commons Attribution (CC BY) license (https:// creativecommons.org/licenses/by/ $4.0 /)$.
Department of Physics and Astronomy, Mississippi State University, Starkville, MS 39762, USA; lge25@msstate.edu (L.E.); pa406@msstate.edu (P.A.)

* Correspondence: pp838@msstate.edu

\begin{abstract}
Fractal dimension, a measure of self-similarity in a structure, is a powerful physical parameter for the characterization of structural property of many partially filled disordered materials. Biological tissues are fractal in nature and reports show a change in self-similarity associated with the progress of cancer, resulting in changes in their fractal dimensions. Here, we report that fractal dimension measurement is a potential technique for the detection of different stages of cancer using transmission optical microscopy. Transmission optical microscopy of a thin tissue sample produces intensity distribution patterns proportional to its refractive index pattern, representing its mass density distribution. We measure fractal dimension detection of different cancer stages and find its universal feature. Many deadly cancers are difficult to detect in their early to different stages due to the hard-to-reach location of the organ and/or lack of symptoms until very late stages. To study these deadly cancers, tissue microarray (TMA) samples containing different stages of cancers are analyzed for pancreatic, breast, colon, and prostate cancers. The fractal dimension method correctly differentiates cancer stages in progressive cancer, raising possibilities for a physics-based accurate diagnosis method for cancer detection.
\end{abstract}

Keywords: fractal dimension; cancer; transmission optical microscopy

\section{Introduction}

An object exhibiting self-similar structures at different length scales is known as a fractal. Several systems in nature are fractal, including biological tissue samples from different organs [1-5]. Biological tissues have spatial heterogeneity in their mass density distribution and are a self-similar structure. This self-similar structure can be analyzed and expressed in terms of fractal dimension. The fractal dimension of an object is a number quantifying how similar the structure remains with changes in length scale and is related to the structural porosity of the tissue samples. It is now known that the fractal dimension of tissue changes with the progress of cancer [6-10]. A tissue's fractal dimension will change throughout the course of cancer progression due to the increased production and rearrangement of intracellular structures such as DNA, RNA, lipids, heterochromatin, and the extracellular matrix, which causes an increase in the mass density and rearrangement of the tissue. Because cancer progression changes a tissue's fractal dimension, a quantitative diagnostic test can be developed based on these changes [11,12]. Therefore, a physics-based quantitative approach that detects structural change with the progression of carcinogenesis, in particular changes in the fractal dimension, at the earliest possible point as well as the time progression of carcinogenesis may be a potential parameter for characterization of the progress of cancer. Although several reports are measuring fractal dimensions for cancer detection, they are mainly individual cancer cases and the topic requires more exploration $[6,10]$.

According to the $\mathrm{WHO}$, millions of people are dying from cancer each year. Therefore, accurate detection of early stages of cancer, as well as different stages of cancer progression, 
is necessary for preventing this widescale mortality [13]. Cancer arises in people due to changes in the DNA replication processes that occur during cell reproduction due to random processes. This may be due to the influence of carcinogenic chemicals, radiation exposure, or many other factors. Cancerous cells tend to reproduce themselves more rapidly than normal cells, causing a massive buildup of abnormal cells known as a tumor. These tumors inhibit the normal bodily functions of the organs they reside in and then can spread through the bloodstream or immune network to several vital organs such as the lungs, brain, liver, etc., resulting in a potentially fatal outcome if left to grow unchecked. Currently, most cancer tests rely on a pathologist qualitatively studying a cell/tissue from a biopsy tissue/cell sample for minute structural differences or they rely on chemical methods involving exceedingly expensive and time-consuming dyes and stains $[14,15]$. Both of these methods are inefficient and potentially inaccurate due to potential bias for the staining chemical and the reliance on the observational abilities of the pathologist. Due to the high potential for human error in eye detection in conventional techniques, a more easy, quantitative, physics-based test is necessary to help detect cancer and its stage characterization.

To test the usefulness of the potential of the fractal method for cancer detection and its stage characterization, as well as to explore its universal approach of applying this method to different cancers, tissue microarray (TMA) samples from common cancer types, pancreatic, breast, colon, and prostate, were utilized. TMA samples are a method of commercially available clinical sample observation that is increasingly growing in popularity, especially for research purposes, that avoids the complicated process of collecting tissue directly from patients in hospitals. TMA consists of a glass slide containing multiple cores of consistent paraffin embedded tissue samples from the same bodily region of several human subjects of control and with varying stages of cancer. In our case, each core consists of a circular sample of tissue that is $1.5 \mathrm{~mm}$ in diameter and $5 \mu \mathrm{m}$ in thickness embedded in paraffin wax to increase the sample's shelf-life. Each slide can contain up to a hundred cores, greatly increasing the speed and high-throughput analysis at which multiple tissue samples can be processed and analyzed [16,17]. For the purpose of the present experiment, four different cancer TMA slides containing only 24 cores each of different cases were used. The following four cancer types were studied using TMA samples: pancreatic, breast, colon, and prostate. Pancreatic cancer was chosen due to its lethality; it is estimated that 48,220 people will die from this disease in the United States in 2021. Its mortality is due in large part to the organ's location being difficult to reach, and because it does not display any prominent chemical changes or symptoms until the hard-to-treat late stages. Another cancer that was studied was breast cancer due to its prevalence. In 2021, it is estimated that over 281,550 women in the United States will be diagnosed with breast cancer, which is characterized by a diverse range of potential causes including radiation exposure, consumption of alcohol, and age at which a first pregnancy occurs $[18,19]$. Colon and prostate cancer, both of which have been linked to high consumption of red meat, also exhibit high rates of mortality and diagnosis rates, so they too were studied using the fractal dimension technique $[18,20,21]$. It is estimated that, in 2021, these four cancers will be responsible for the deaths of almost 180,000 people within the United States alone [18]. Due to either their prevalence, lethality, or combination of the two, pancreatic, breast, colon, and prostate cancers were analyzed using this quantitative method, which has the potential to become a new diagnosis method.

The key concepts explored in this paper are as follows: (1) the mass density structure of the tissue is fractal, which is self-similar in all length scales and has a fractal dimension. (2) The progress of cancer is associated with more mass density accumulation in the tissue and in turn leads to changes in the fractal dimension of the tissue. (3) The refractive index of tissue is proportional to its mass density; therefore, change in the tissue due to cancer progression can be probed by the optics. (4) In this study, we quantify the different stages of cancer in tissue by calculating the fractal dimension of this cancerous tissue by probing the tissue structure by a bright field transmission microscope. A flow chart for the steps are shown in Figure 1. 


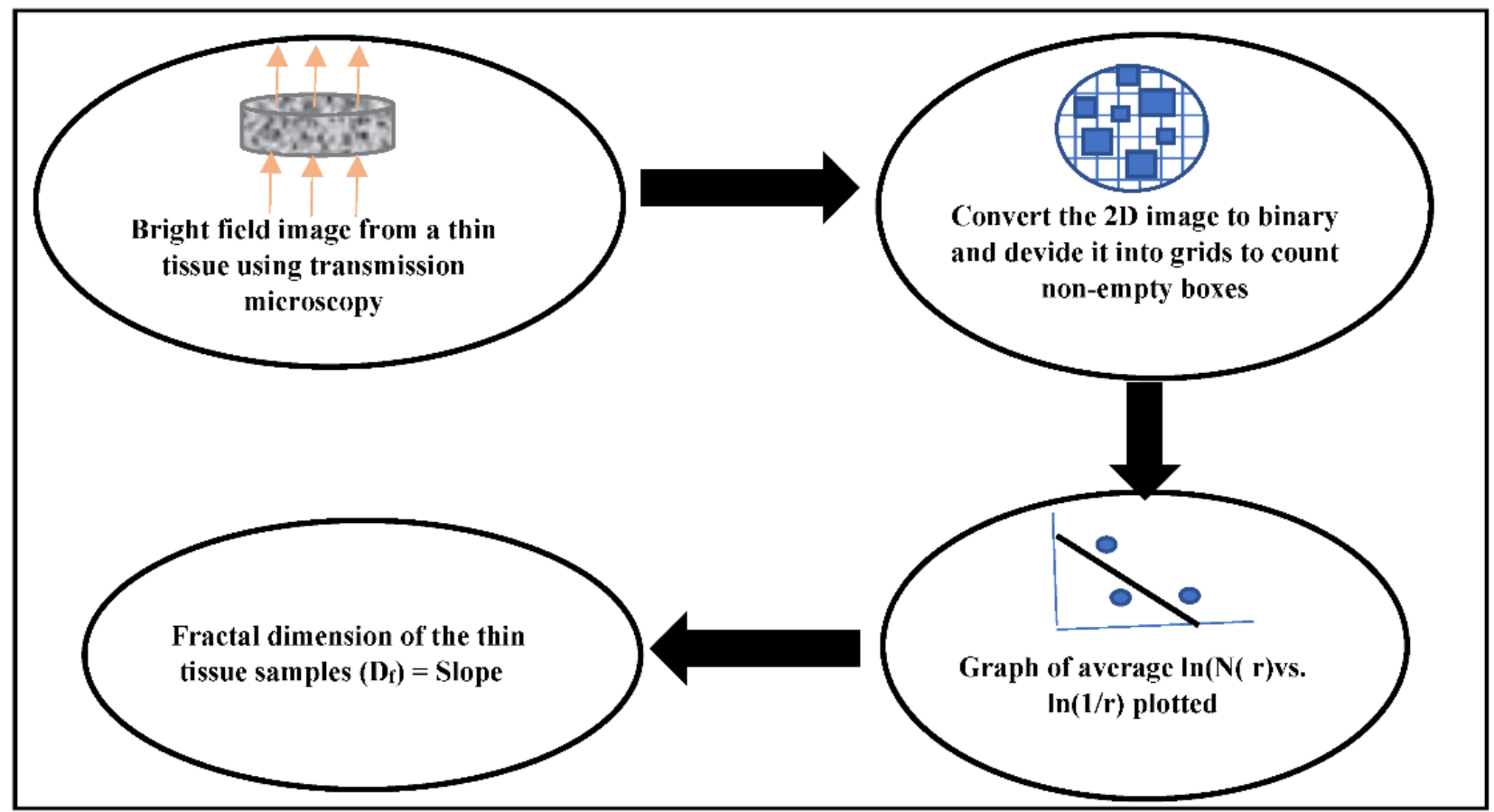

Figure 1. The step-by-step process for calculating the fractal dimension of a microscopic image is outlined.

\section{Materials and Methods}

\subsection{Materials}

Human tissue samples are obtained commercially from US Biomax Inc. (Derwood, MD, USA), that has collected the tissue samples with the legal and ethical procedure (as documents provided by the Biomax).

\subsection{Mathematical Methods}

\subsubsection{Fractals and Fractal Dimensions}

Fractals are samples with partially filled structures in space that exhibit self-similarity at different length scales and can be divided into two categories: deterministic and random. Deterministic fractals are generated deliberately by systematically removing or filling a sample with a deterministic algorithm, while random fractals are created through a stochastic process [22]. Random fractals occur quite often in nature and can be seen in the structures of trees, coastlines, and most importantly for this experiment, in tissue structures. These random fractals can have varying degrees of self-similarity, which leads to the need for a quantifiable fractal dimension. A structure's fractal dimension is essentially a measure of how self-similar the structure is; the higher the dimension, the more self-similar it is, with more filling or less porosity. The fractal dimension of a random fractal is determined using a box-counting method. This method works by first placing the fractal structure on an evenly spaced grid and counting the number of boxes needed to cover the structure [23,24]. According to the definition of a fractal object, it follows the relationship of different length scales with the following mathematical relations between any two length scales: $N_{i}\left(r_{i}\right) \times\left(r_{i}\right)^{D f}=N_{j} \times\left(r_{j}\right)^{D f}=$ Constant, where $N_{i}$ is the number of boxes at length scale $r_{i}$, and $N_{j}$ is the number of boxes at length scale $r_{j}$. The average fractal dimension $D_{f}$ is then calculated by refining the grid through a box-counting algorithm. The equation used in this algorithm can be derived from the above definition as an average slope of $\ln (N(r))$ vs. $r$ curve, with varying length scales $r$.

$$
D_{f}=\frac{\ln (N(r))}{\ln \left(\frac{1}{r}\right)}
$$


where $N(r)$ is the number of boxes of side length $r$ needed to cover the fractal structure. $\mathrm{D}_{f}$ is the ensemble averaged fractal dimension of the structure since the box-counting method accounts for several different realizations.

\subsubsection{Calculating Fractal Dimension using Microscopic Images}

We report here the study of fractal dimension from paraffin embedded thin tissue samples and attempt to see the universality in the change in the fractal dimensions for most common deadly cancer cases. Some aspects of this method have been utilized in previous studies of cancer detection [14,25]. This method works by taking the binary version of an image and applying the box-counting method to divide the image into a grid of evenly sized boxes and creating a plot of $\ln (N(r))$ vs. $\ln (1 / r)$ whose average slope is equal to the fractal dimension. While most of the previous experiments focused on a single type of cancer, the experimental results reported in this paper deals with multiple types of cancer to explore the potential of creating a standardized numerical index that applies to many cancers and to explore their universality with less intensity tissue samples (paraffin embedded), and lastly, to see any universality.

\subsection{Physical Set-Up}

\subsubsection{TMA Samples}

Four different TMA samples from US Biomax were studied in this experiment: pancreatic (T142b), breast (BR248a), colon (T054c), and prostate (T191a). Each slide consists of around 24 cores of $1.5 \mathrm{~mm}$ in diameter and $5 \mu \mathrm{m}$-thick tissue that were embedded in paraffin wax and attached to the slide. For each sample, at least 3-5 cores of each stage of control or cancer are analyzed. Each core on the pancreatic and colon TMA slides comes from individual patients of varying ages and sex. The prostate TMA slide contains 12 pairs of identical cores, where each pair was obtained from a different patient. The breast TMA slide contains individual cores from female patients of varying ages of control and cancer cases. These cores were either normal tissue samples or tissue samples in stage I, stage II, or stage III of cancer.

\subsubsection{Microscope Setup and Imaging}

The images were taken using an Olympus BX61 Upright Bright field Microscope (Tokyo, Japan) coupled with an Amscope model MU1003 camera using Amscope software. Each TMA slide is imaged on the microscope stage, which is being operated in brightfield transmission. At least 20-22 different cores are imaged on each slide, depending on the number of cores and stages of cancer present. Each core is imaged with $50 \times$ magnification objectives without immersion oil in at least 10 different spots. Every spot has 5-10 images taken at slightly different focusing heights to ensure at least one focused image is captured within the working distance of the objective. The best focus map was obtained by the most intense average gray spots.

For every biological sample, change in certain/overall refractive index properties can be attributed to the progress of cancer. In the early stages of cancer, there is an accumulation of nucleic acid in the nuclei followed by unregulated cell division in the advanced stages of cancer. These properties show a distinct change when cancer present in the sample causes a small linear increase in the sample's mass density [26]. In particular, an increase in the mass density and filling of the porosity or less mass density areas happens with the progress of cancer. For this study, the contrast of the grayscale images taken with the microscope is assumed to be caused by the spatial mass density variation within the sample. This variation provides the refractive index variation, which, in turn, provides the correlation between the mass density of the sample and pixel intensity/transmission intensity of the image. 
Transmission intensity $I_{t}$ of a biological tissue sitting on a glass slide of an incident intensity $I_{0}$ can be expressed as the transmission through a thick sample, and it follows the following equations:

$$
I_{t}=I_{0} \times\left(1-\frac{n_{\text {tissue }+ \text { glass }}-n_{\text {air }}}{n_{\text {tissue }+ \text { glass }}+n_{\text {air }}}\right) \times \exp ^{(-K . d)}
$$

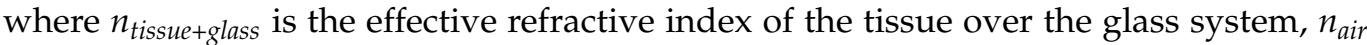
is the refractive index of the air, $K$ is the effective absorption coefficient of the tissue over the glass, and $d$ is the thickness of the slide with the tissue sample. For a TMA tissue sample, $I_{0}, K$, and $d$ are constants (for dried tissue, the absorption is quite negligible), and there is a linear relationship between transmission intensity and change in the refractive index $\left(n_{\text {tissue }}\right.$ glass $\left.-n_{\text {air }}\right)$.

$$
I_{t} \alpha\left(n_{\text {tissue }+ \text { glass }}-n_{\text {air }}\right) \alpha \text { change in tissue mass density }
$$

This implies the change in image intensity pattern is proportional to the change in the refractive index of the tissue, where the refractive index is proportional to the mass density.

\subsection{Analysis of Images}

Each captured microscopy image is analyzed using Image J (NIH, Bethesda, MD, USA) software [27]. First, the bright-field images are converted into 8-bit binary images with Image $J$. The threshold for determining the background versus objects in the image is calculated automatically by Image J by first taking a test threshold, computing the average pixels above and below the threshold, and then incrementing the threshold. This process is repeated until the threshold is larger than the composite average as described in the equation.

$$
\text { Threshold }=(\text { average background }+ \text { average objects }) / 2
$$

The microscopy best focusing was performed by moving the focusing around the best focus point and then performing a histogram of best mean gray points. After the image is converted to binary, a smaller area is selected for each realization, and its surroundings are cropped to remove any dead space or deformities in the tissue structure of the TMA sample. Finally, the fractal dimension is calculated using the box-counting method, which is performed by the fractal analysis through the method mentioned above in Section 2.2.2.

\subsection{Statistical Analysis}

All the statistical analyses of the calculation of the p-value for relative changes in the fractal dimension were performed using Student's $t$-test (two-tailed).

\section{Results}

After collecting microscopic images of each sample, they are converted into binary images in Image J. Following that, the fractal dimension analysis tool in Image $J$ is used to calculate the fractal dimension of each cancer stage of TMA samples, which are then graphed for comparison. As seen in the graphs, the fractal dimension for the pancreatic, breast, colon, and prostate cancer samples increases with the progression of cancer through the different stages. This increase in fractal dimension is because the presence of cancer results in a higher cell replication rate [28]. The rate of cancerous cell replication is greater than that of normal cells due to genetic mutations. These genetic mutations also cause the cancerous cells to spread to other regions of the body at a higher rate than the normal cells [29]. As the cells reproduce at a faster rate, the mass density of the affected tissue area increases. Since the fractal dimension of a tissue sample is dependent on this mass density, it will increase as the mass density increases due to the progression of cancer. 


\subsection{Pancreatic Cancer}

Figure $2 \mathrm{a}, \mathrm{b}$ show the brightfield images of normal and stage III pancreatic tissue from a TMA sample, respectively. Figure $2 a^{\prime}, b^{\prime}$ show the binary versions of each brightfield images. These binary images display the increased mass density distribution, which results from the progression of cancer. As seen in Figure 2c, the normal pancreatic tissue sample had the lowest fractal dimension, while the different stages of cancer possess increasing fractal dimensions. The actual fractal dimensions for each sample are calculated to be 1.5984 for the normal, 1.6673 for stage I, 1.6866 for stage II, and 1.7407 for stage III. The percent difference between the normal and stage I sample is $4 \%$, between normal and stage II is $6 \%$, and between normal and stage III is $9 \%$. This percentage increment in the logarithm scale of the fractal dimension with the increase in the stage of cancer is highly significant. These results suggest that the fractal dimension of a pancreatic tissue TMA sample increases with the progression of cancer. This is a logical outcome, as cancer is known to cause cell replication to increase, which then causes the density, and therefore the self-similarity, of cells within the tissue to increase as mentioned earlier. This results in variation to the transmission intensities from the sample, which increases the fractal dimension. Since the fractal dimension is a measure of the self-similarity of a sample, the fractal dimension of pancreatic tissue will increase with the progress of cancer. Similar results have also been observed in several other studies [30].
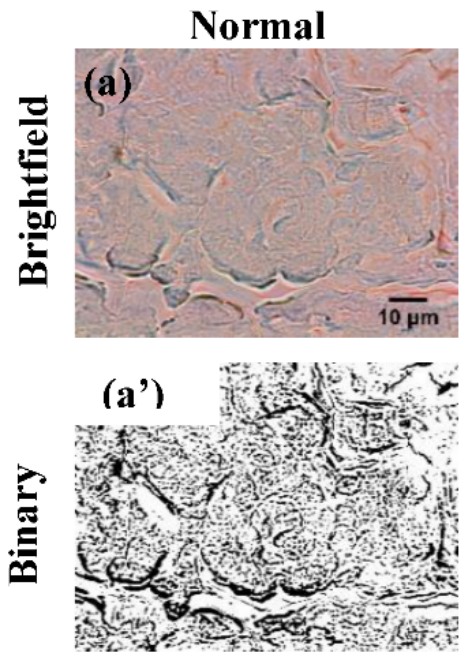
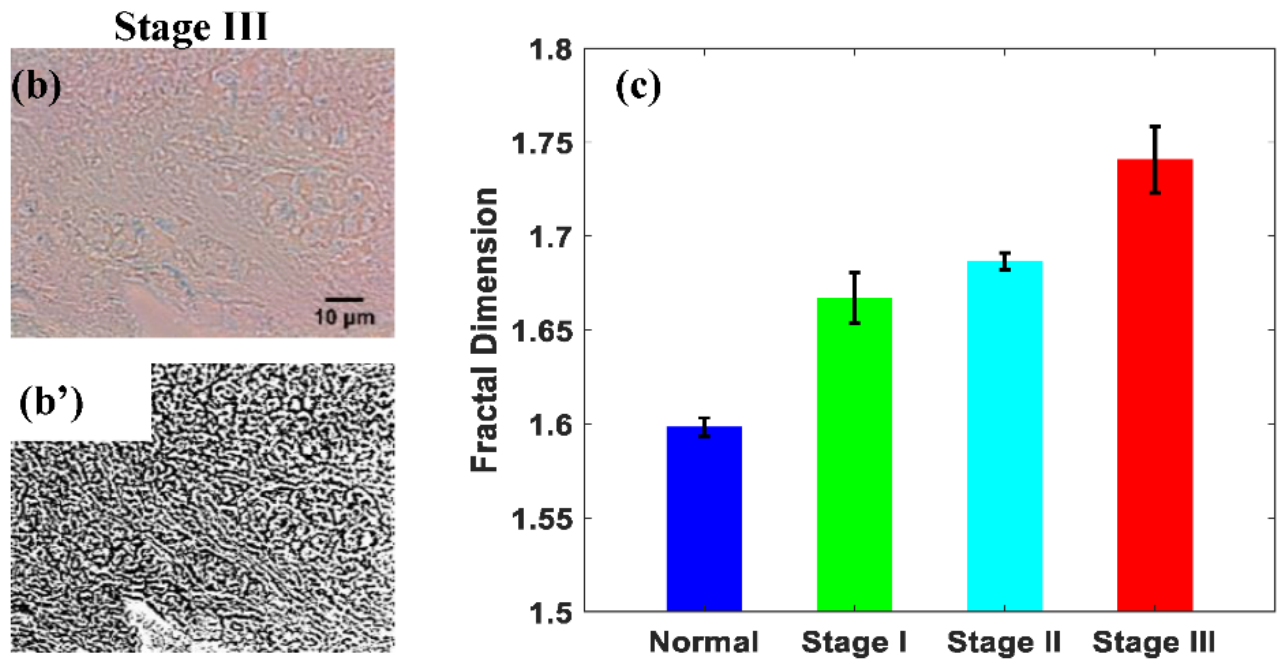

Figure 2. Pancreatic cancer: $(\mathbf{a}, \mathbf{b})$ are the brightfield images of the normal and stage III pancreatic TMA. $\left(\mathbf{a}^{\prime}, \mathbf{b}^{\prime}\right)$ are the corresponding binary images. (c) is the bar graph of the average fractal dimension of the pancreatic tissue samples ( $n=24,3-5$ subjects per stage). The results show the fractal dimension of cancer stage I increases by $4 \%$, stage II by $6 \%$, and stage III by $9 \%$ with respect to the normal ( $p$-values < 0.05).

\subsection{Breast Cancer}

The brightfield images from a normal and stage III breast cancer TMA tissue core are shown in Figure 3a,b, respectively. Their binary analogs are shown in Figure 3a', $b^{\prime}$, with Figure $3 b^{\prime}$ showing an increased density. The greater density demonstrates how the progression of cancer results in an increased mass density distribution. As shown in Figure 3c, the normal breast tissue sample has the lowest fractal dimension, while the different stages of cancer had fractal dimensions increasing with the progression of cancer. The actual fractal dimensions of the breast tissue samples are 1.5448 for the normal, 1.6126 for stage I, 1.6631 for stage II, and 1.7284 for stage III cancer. For the normal, and stage I tissue samples, the percent difference between the two is $4 \%$, while the percent difference between the normal and stage II and the normal and stage III are $7 \%$ and $12 \%$, respectively. These results suggest that the fractal dimension of a breast TMA sample increases with the progression of cancer. Cancer is known to cause an increase in the 
replication rate of cells, which therefore causes an increase in the tissue's mass density distribution. These results make sense because the fractal dimension of a tissue sample is a measure of its mass density distribution. Similar results to these have also been observed in other studies [30].
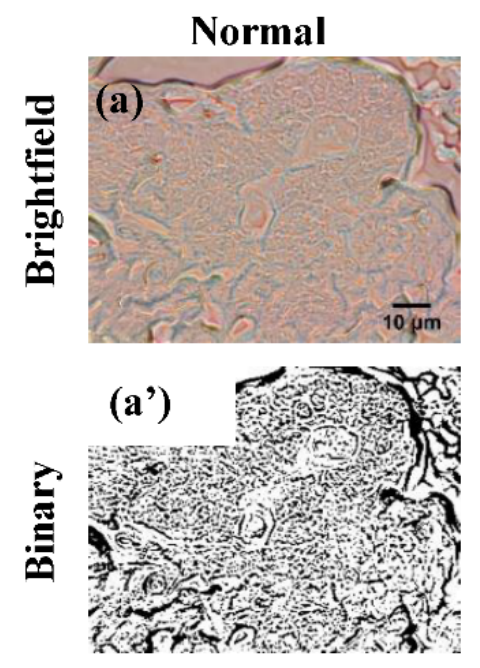

\section{Stage III}

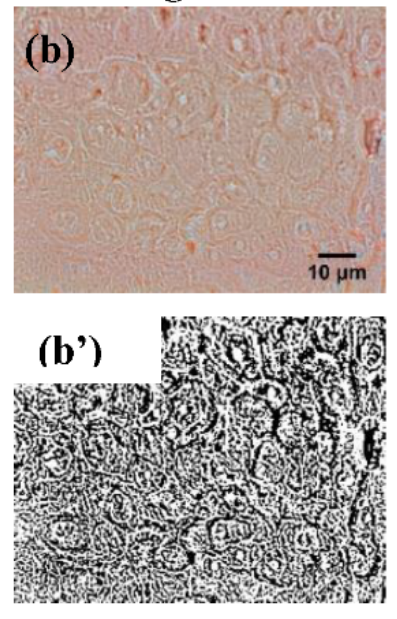

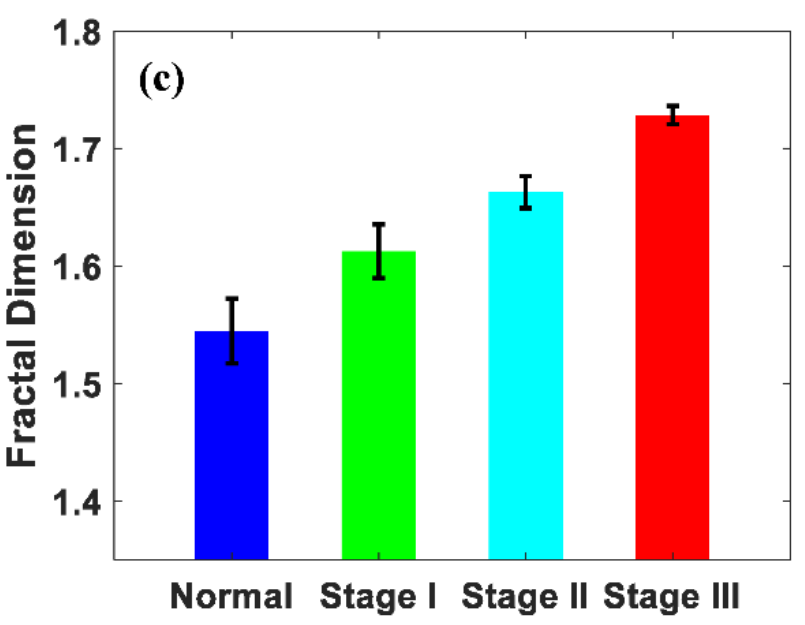

Figure 3. Breast cancer: $(\mathbf{a}, \mathbf{b})$ are the brightfield images of the normal and stage III Breast TMA. $\left(\mathbf{a}^{\prime}, \mathbf{b}^{\prime}\right)$ are the corresponding binary images. (c) is the bar graph of the average fractal dimension of the Breast cancer tissue microarrays (TMA) samples ( $n=24,3-5$ subjects per stage). The results show the fractal dimension of cancer stage I increases by $4 \%$, stage II by $7 \%$, and stage III by $12 \%$ with respect to the normal ( $p$-values $<0.05)$.

\subsection{Colon Cancer}

Normal and stage III cores of the colon TMA slide's brightfield images are shown in Figure $4 a$, b. Their corresponding binary images are pictured in Figure $4 a^{\prime}, b^{\prime}$ and show how the mass density distribution of a tissue sample increases throughout the progression of cancer. As can be seen in Figure 4c, the normal colon tissue sample possesses the lowest fractal dimension, while the fractal dimensions for each stage of cancer increase with its progression. The actual fractal dimension values for each sample are 1.5551 for the normal, 1.6393 for stage I, 1.6652 for stage II, and 1.7004 for stage III. When the normal tissue sample is compared with stage I, stage II, and stage III samples, the percent differences are found to be $5 \%, 7 \%$, and $9 \%$, respectively. These results suggest that the fractal dimension of a colon TMA sample increases with the progression of cancer. Like other tissue samples, the presence of colon cancer causes the replication rate of the affected cells to increase, resulting in an increased mass density distribution. The fractal dimension of the tissue sample will increase due to this, so the results gathered from this study make sense. Similar results have also been observed in other studies [18].

\subsection{Prostate Cancer}

Representative brightfield images of a normal and stage III core are presented in Figure $5 a, b$, respectively. Figure $5 a^{\prime}, b^{\prime}$ are the binary versions of the brightfield images obtained through the Image J software. They clearly show that the mass density distribution within tissue increases with the progression of cancer. As seen in Figure $5 c$, the normal prostate tissue possesses the lowest fractal dimension, while the fractal dimensions for each stage of cancer increase with its progression. The actual fractal dimension values for each sample are 1.5737 for the normal, 1.5981 for stage I, 1.6302 for stage II, and 1.6798 for stage III. The percent difference between the normal and stage I samples is $2 \%$, normal and stage II samples is $4 \%$, and the difference between the normal and stage III is $7 \%$. These results suggest that the fractal dimension of a prostate TMA sample increases with the progression 
of cancer. The mass density distribution of a tissue sample increases due to the increased cell replication caused by the presence of cancer, supporting the results obtained [25,31,32].
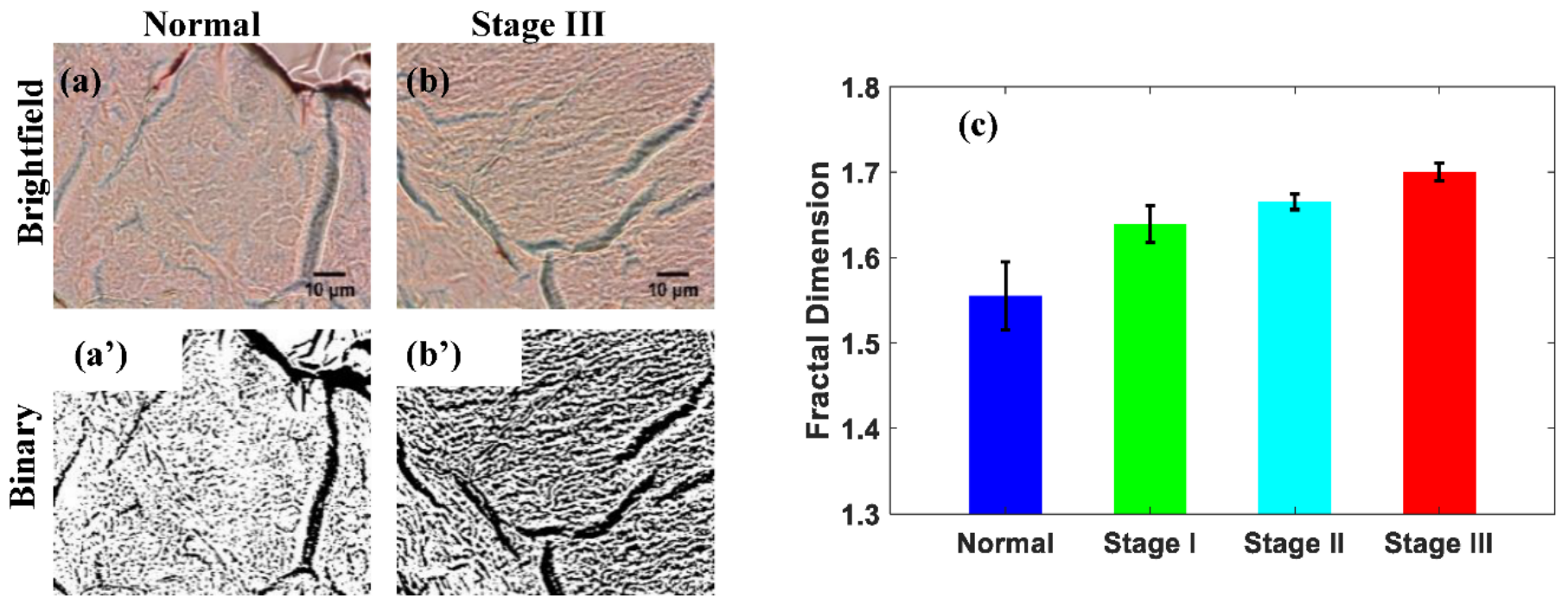

Figure 4. Colon cancer: $(\mathbf{a}, \mathbf{b})$ are the brightfield images of the normal and stage III Colon TMA. $\left(\mathbf{a}^{\prime}, \mathbf{b}^{\prime}\right)$ are the corresponding binary images. (c) is the bar graph of the average fractal dimension of the Colon cancer tissue microarrays (TMA) samples $(n=24,3-5$ subjects per stage). The results show the fractal dimension of cancer stage I increases by $5 \%$, stage II by $7 \%$, and stage III by $9 \%$ with respect to the normal ( $p$-values $<0.05)$.
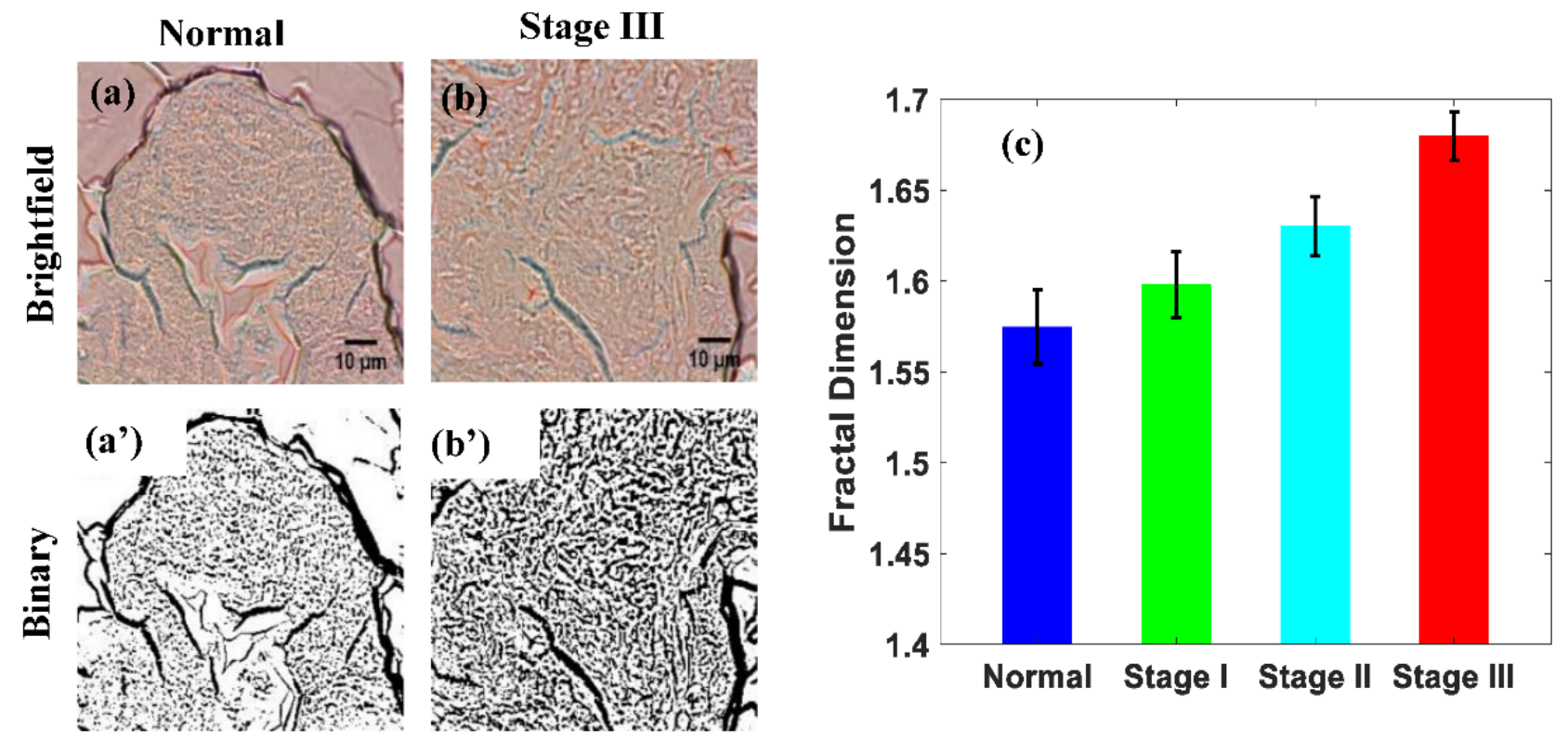

Figure 5. Prostate cancer: $(\mathbf{a}, \mathbf{b})$ are the brightfield images of the normal and stage III Prostate TMA. $\left(\mathbf{a}^{\prime}, \mathbf{b}^{\prime}\right)$ are the corresponding binary images. (c) is the bar graph of the average fractal dimension of the prostate cancer tissue microarrays (TMA) samples ( $\mathrm{n}=24,3-5$ subjects per stage). The results show the fractal dimension of cancer stage I increases by $2 \%$, stage II increases by $4 \%$, and stage III by $7 \%$ with respect to the normal ( $p$-values $<0.05)$.

\section{Discussions}

In this section, we compare the variation of different stages of cancer with respect to the normal for the four main cancer cases considered here: pancreatic, breast, colon, and prostate. The actual value of the fractal dimensions of the tissue with the increase in cancer 
stages has presented the by the Table 1 for better clarification. Also, the average values of fractal dimension of the different stages of different cancer are graphically represented in Figure 6, which concludes the universalities of fractal dimension with the increase in cancer stages.

Table 1. The mean values of the fractal dimension of different cancer for different stages.

\begin{tabular}{ccccc}
\hline & Normal & Stage I & Stage II & Stage III \\
\hline Pancreatic & 1.5984 & 1.6673 & 1.6866 & 1.7047 \\
\hline Breast & 1.5448 & 1.6126 & 1.6631 & 1.7283 \\
\hline Colon & 1.5551 & 1.6393 & 1.6652 & 1.7004 \\
\hline Prostate & 1.5737 & 1.5981 & 1.6302 & 1.6798 \\
\hline
\end{tabular}

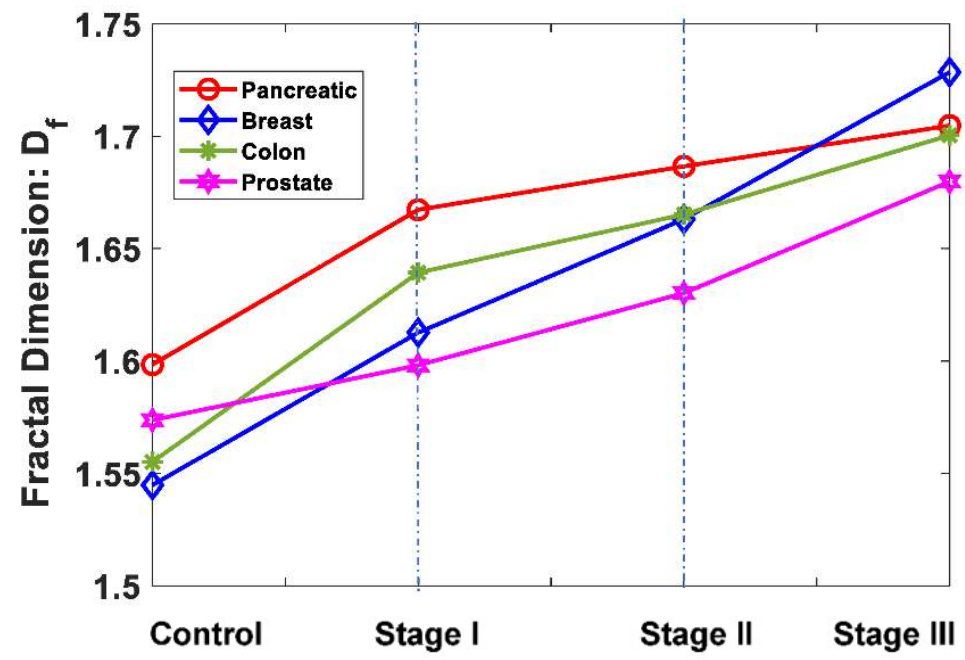

Figure 6. The plot of the systematic increases in the fractal dimension with the progress of the cancer stages. The $x$-axis steps are false steps that are measured by an equal distance unit of 1 for increasing stages, for better presentation. It can be seen that the fractal dimension on average increases with the stage; however, they take slightly different paths. Note: only mean values are shown, standard errors are the same as shown in bar graph plots.

From the above data, the universal nature of the fractal dimension increasing with the progress of cancer is seen; that is, there is an increase in the fractal dimension from normal to Stage I to Stage II to Stage III. However, the paths have slight differences for different types of cancers. Hypothetically, when there will be no space left for filling as in a very advanced stage of the cancers, all cancer types will have a fractal dimension of two (for the 2D tissue samples) and will meet at the same point.

It can be pointed out here that, recently, there have been several studies of cells and tissues where the fractal dimension calculations have been explored using lab-developed quantitative phase microscopies, where mass structures can be detected, and fractal dimensions can be calculated. However, the present paper addresses the most basic bright-field transmission optical microscopy for fractal dimension calculation, which is available to most biomedical laboratories or hospitals. A combination of bright field transmission microscopy and quantitative phase microscopy may provide more accurate fractal dimensions or more accurate stages of cancer detection that need to be explored [33-36].

\section{Conclusions}

In this paper, we explored the possibility of creating a standardized, physics-based cancer diagnosis test capable of accurate detection of cancer in both early and late stages based on the fractal dimension analysis of paraffin embedded TMA samples. This was 
undertaken by optical transmission microscopic imaging of TMA samples of different stages of pancreatic, breast, colon, and prostate cancer and calculating their fractal dimensions. Intensity distribution from a thin tissue sample using transmission bright field microscopy is directly related to the mass density distribution of the tissue, and the fractal dimensions are calculated. The results show that in all the four types of cancer cases studied here, the mean fractal dimension of a tissue sample increased as cancer progresses through the different stages, with slightly different paths. The fractal dimension increases due to the increase in the mass density and filling of the tissue porosity structure with the progress of cancer. These results support that the histogram of the grayscale average represents the degree of tissue disorganization, which reduces with the progression of carcinogenesis. Therefore, the normal of each cancer tissue has a minuscule gray scale, while the higher stages of each cancer have a higher grayscale or fractal dimension due to more accumulation of mass density, such as due to the higher accumulation of DNA mass, etc. Here, we were able to distinguish the different stages of pancreatic, breast, colon, and prostate cancer cases via computer-aided/automated quantitative approach of the fractal dimension detection of commercially available TMA samples using optical transmission imaging. This result shows that there is definite potential for the creation of a physics-based, standardized, automated, and quantitative cancer diagnosis approach, which is free of some of the issues plaguing the current cancer diagnosis methods that are based on the eye estimation of pathologists. Lastly, a physics-based easy, prompt, cost-effective, and accurate quantitative cancer diagnosis method can also be compiled into a database with the possibility for the creation of a shared database accessible to every physician. By compiling the fractal dimensions that are calculated by doctors all over the country, a master list can be created that gives the range of the average fractal dimension for each type and stage of cancer and helps to distinguish the early stages of cancer. This master list would become increasingly more accurate as more data is entered and be further improved based on this structural change detection method of fractal dimension for early cancer and its progression of different stages. We have to note here that, because the fractal dimension changes logarithmically with the filling, this makes fractal dimension change slow but it is still a powerful parameter for characterization of the progression of cancer and its different stages.

Author Contributions: P.P. designed the project; L.E. perform the experiments; L.E. and P.P. perform the data analyses; P.A. helped in experiments; L.E., P.A. and P.P. wrote the main paper. All authors have read and agreed to the published version of the manuscript.

Funding: This research was funded by the National Institute of Health (Grant No. R21CA260147) and ORED (Mississippi State University).

Data Availability Statement: Data available on request.

Acknowledgments: The work was partially supported by the National Institute of Health (Grant No. R21CA260147) and ORED, Mississippi State University support for research.

Conflicts of Interest: The authors declare no conflict of interest.

\section{References}

1. Theiler, J. Estimating fractal dimension. JOSA A 1990, 7, 1055-1073. [CrossRef]

2. Fernández-Martínez, M.; Sánchez-Granero, M.A. Fractal dimension for fractal structures. Topol. Its Appl. $2014,163,93-111$. [CrossRef]

3. Losa, G.A.; Merlini, D.; Nonnenmacher, T.F.; Weibel, E.R. Fractals in Biology and Medicine: III; Springer Science \& Business Media: Berlin/Heidelberg, Germany, 1994; Volume 3.

4. Fielding, A. Applications of fractal geometry to biology. Bioinformatics 1992, 8, 359-366. [CrossRef] [PubMed]

5. Losa, G.A.; Nonnenmacher, T.F. Self-similarity and fractal irregularity in pathologic tissues. Mod. Pathol. 1996, 9, 174-182. [PubMed]

6. $\quad$ Davies, N.A.; Harrison, N.K.; Morris, R.H.K.; Noble, S.; Lawrence, M.J.; D'Silva, L.A.; Evans, P.A. Fractal dimension (df) as a new structural biomarker of clot microstructure in different stages of lung cancer. Thromb. Haemost. 2015, 114, 1251-1259. [CrossRef] 
7. Etehad Tavakol, M.; Lucas, C.; Sadri, S.; Ng, E.Y.K. Analysis of breast thermography using fractal dimension to establish possible difference between malignant and benign patterns. J. Healthc. Eng. 2010, 1, 27-43. [CrossRef]

8. Huang, P.W.; Lee, C.H. Automatic classification for pathological prostate images based on fractal analysis. IEEE Trans. Med. Imaging 2009, 28, 1037-1050. [CrossRef] [PubMed]

9. Chan, A.; Tuszynski, J.A. Automatic prediction of tumor malignancy in breast cancer with fractal dimension. R. Soc. Open Sci. 2016, 3, 160558. [CrossRef]

10. Uthayakumar, R.; Jayalalitha, G. Border detection of skin cancer cells with fractal dimension. Fractals 2009, 17, 171-180. [CrossRef]

11. Bhandari, S.; Choudannavar, S.; Avery, E.R.; Sahay, P.; Pradhan, P. Detection of colon cancer stages via fractal dimension analysis of optical transmission imaging of tissue microarrays (TMA). Biomed. Phys. Eng. Exp. 2018, 4, 065020. [CrossRef]

12. Adhikari, P.; Binu, A.P.; Bhandari, S.; Khan, S.; Jaggi, M.; Chauhan, S.C. Optical Detection of Fractal Dimensions of MUC13 Stained Pancreatic Tissues for Cancer Diagnostics. arXiv 2018, arXiv:181210883.

13. Wulfkuhle, J.D.; Liotta, L.A.; Petricoin, E.F. Proteomic applications for the early detection of cancer. Nat. Rev. Cancer 2003, 3 , 267-275. [CrossRef] [PubMed]

14. Loeb, L.A.; Springgate, C.F.; Battula, N. Errors in DNA Replication as a Basis of Malignant Changes. Cancer Res. 1974, 34, 2311-2321.

15. Lingen, M.W.; Kalmar, J.R.; Karrison, T.; Speight, P.M. Critical evaluation of diagnostic aids for the detection of oral cancer. Oral Oncol. 2007, 44, 10-22. [CrossRef]

16. Shergill, M.I.S.; Shergill, N.K.; Arya, M.; Patel, H.R.H. Tissue microarrays: A current medical research tool. Curr. Med. Res. Opin. 2004, 20, 707-712. [CrossRef]

17. Jawhar Nazar, M.T. Tissue Microarray: A rapidly evolving diagnostic and research tool. Ann. Saudi Med. 2009, 29, 123-127. [CrossRef] [PubMed]

18. Cancer Statistics. 2019-Siegel-2019-CA: A Cancer Journal for Clinicians-Wiley Online Library [Internet]. Available online: https:/ / onlinelibrary.wiley.com/doi/full/10.3322/caac.21551 (accessed on 30 October 2019).

19. MacMahon, B. Epidemiology and the causes of breast cancer. Int. J. Cancer 2006, 118, 2373-2378. [CrossRef] [PubMed]

20. Giovannucci, E. Epidemiologic Characteristics of Prostate Cancer. Cancer 1995, 75, 1766-1777. [CrossRef]

21. Giovannucci, E. Willett WC. Dietary Factors and Risk of Colon Cancer. Ann. Med. 1994, 26, 443-452. [CrossRef]

22. Bunde, A.; Havlin, S. Fractals in Science; Springer: Berlin/Heidelberg, Germany, 2013; 317p.

23. Li, J.; Du, Q.; Sun, C. An improved box-counting method for image fractal dimension estimation. Pattern Recognit. 2009, 42, 2460-2469. [CrossRef]

24. Panigrahy, C.; Seal, A.; Mahato, N.K. Quantitative texture measurement of gray-scale images: Fractal dimension using an improved differential box counting method. Measurement 2019, 147, 106859. [CrossRef]

25. de Arruda, P.F.F.; Gatti, M.; Junior, F.N.F.; de Arruda, J.G.F.; Moreira, R.D.; Murta, L.O. Quantification of fractal dimension and Shannon's entropy in histological diagnosis of prostate cancer. BMC Clin. Pathol. 2013, 13, 6. [CrossRef]

26. Davies, H.G.; Wilkins, M.H.F. Interference Microscopy and Mass Determination. Nature 1952, 169, 541. [CrossRef]

27. Image-j. Available online: https://imagej.nih.gov/ij/ (accessed on 1 November 2021).

28. Preston-Martin, S.; Pike, M.C.; Ross, R.K.; Henderson, B.E. Epidemiologic evidence for the increased cell proliferation model of carcinogenesis. Environ. Health Perspect 1993, 101 (Suppl. 5), 137-138. [PubMed]

29. Sahai, E. Mechanisms of cancer cell invasion. Curr. Opin. Genet. Dev. 2005, 15, 87-96. [CrossRef] [PubMed]

30. Scampicchio, A.; Tura, A.; Sbrignadello, S.; Grizzi, F.; Fiorino, S.; Blandamura, S. Assessment of the Fractal Dimension of Images Derived by Biopsy of Pancreatic Tissue: Implications for Tumor Diagnosis. In Proceedings of the XIV Mediterranean Conference on Medical and Biological Engineering and Computing 2016, Paphos, Cyprus, 31 March-2 April 2016; Kyriacou, E., Christofides, S., Pattichis, C.S., Eds.; Springer International Publishing: Berlin/Heidelberg, Germany, 2016; pp. 393-398.

31. Losa, G.A.; Castelli, C. Nuclear patterns of human breast cancer cells during apoptosis: Characterisation by fractal dimension and co-occurrence matrix statistics. Cell Tissue Res. 2005, 322, 257-267. [CrossRef]

32. Krasowska, M.; Grzywna, Z.J.; Mycielska, M.E.; Djamgoz, M.B.A. Patterning of endocytic vesicles and its control by voltage-gated $\mathrm{Na}+$ channel activity in rat prostate cancer cells: Fractal analyses. Eur. Biophys. J. 2004, 33, 535-542. [CrossRef] [PubMed]

33. Park, Y.; Depeursinge, C.; Popescu, G. Quantitative phase imaging in biomedicine. Nat. Photonics 2018, 12, 578-589. [CrossRef]

34. Kastl, L.; Isbach, M.; Dirksen, D.; Schnekenburger, J.; Kemper, B. Quantitative phase imaging for cell culture quality control. Cytom. Part A 2017, 91, 470-481. [CrossRef] [PubMed]

35. Majeed, H.; Sridharan, S.; Mir, M.; Ma, L.; Min, E.; Jung, W.; Popescu, G. Quantitative phase imaging for medical diagnosis. J. Biophotonics 2017, 10, 177-205. [CrossRef] [PubMed]

36. Wang, Z.; Popescu, G.; Tangella, K.V.; Balla, A. Tissue refractive index as marker of disease. J. Biomed. Opt. 2011, 16, 116017. [CrossRef] [PubMed] 